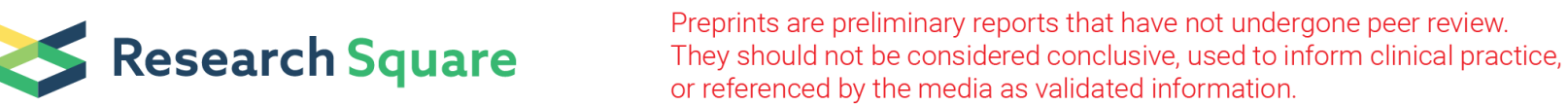

\section{Prognostic Role of Tumor Subtype and Germline BRCA Mutation in Advanced Breast Cancer Patients Treated with Palbociclib Plus Endocrine Therapy}

\section{Song Yi Park}

Seoul National University Hospital Department of Internal Medicine

Koung Jin Suh

Seoul National University Bundang Hospital

Dae-Won Lee

Seoul National University Hospital Department of Internal Medicine

Han Suk Ryu

Seoul National University College of Medicine

Miso Kim

Seoul National University Hospital Department of Internal Medicine

\section{Se Hyun Kim}

Seoul National University Bundang Hospital

\section{Kyung-Hun Lee}

Seoul National University Hospital Department of Internal Medicine

\section{Tae-Yong Kim}

Seoul National University Hospital Department of Internal Medicine Jee Hyun Kim

Seoul National University Bundang Hospital

In Ae Park

Seoul National University College of Medicine

Seock-Ah Im ( $\square$ moisa@snu.ac.kr)

Seoul National University College of Medicine https://orcid.org/0000-0002-5396-6533

\section{Research article}

Keywords: Hormone receptor positive breast cancer, palbociclib, luminal type, BRCA, young Asian breast cancer

Posted Date: August 3rd, 2021

DOI: https://doi.org/10.21203/rs.3.rs-691303/v1 
License: (c) (i) This work is licensed under a Creative Commons Attribution 4.0 International License. Read Full License 


\section{Abstract}

Background/Aims: Palbociclib is a cyclin dependent kinase 4 and 6 inhibitor which shows promising effect in hormone receptor positive breast cancer. The purpose of this study is to evaluate the real-world efficacy and toxicity of palbociclib plus endocrine therapy.

Methods: This is a retrospective study performed in two tertiary referral hospitals in Korea. Advanced breast cancer patients who were treated with 1 st line palbociclib plus endocrine therapy were enrolled.

Results: A total of 216 patients were included between August 2016 and May 2019. Median age was 56 (29-89) years old and 75 patients (34.7\%) were premenopausal. Median progression-free survival (PFS) was 33.0 months (95\% confidence interval [CI] 24.7 to 41.3 ) and objective response rate was $59.3 \%$. Luminal B patients had shorter PFS (Not reached $v s .33 .0$ months, $p=0.019$ ) and tendency of lower ORR (58.3 vs. $62.0 \%, p=0.19$ ) compared to luminal A patients. Multivariate analysis revealed luminal $B$ (adjusted hazard ratio 1.90, $p=0.038$ ) and germline BRCA mutation (adjusted hazard ratio 5.57, $p=$ 0.002 ) as an independent poor prognostic factor for PFS. The most common grade 3 or 4 adverse event was neutropenia (86.7\%).

Conclusions: The efficacy and toxicity of palbociclib in the real-world was comparable to those of clinical trials. In addition, palbociclib with endocrine therapy was an effective treatment option for young patients. Luminal $\mathrm{B}$ and germline BRCA mutation was associated with inferior outcome.

\section{Background}

Breast cancer is the most commonly diagnosed cancer and is the leading cause of cancer death in women $[1,2]$. The incidence rate of breast cancer is increasing annually in developing countries, which is probably due to nation-wide cancer screening and westernization of lifestyle [3, 4]. Up to $75 \%$ of breast cancers express hormone receptor (HR) and endocrine therapy is the main treatment option for these patients [5].

Recently, cyclin dependent kinase (CDK) 4 and 6 inhibitors have emerged as a new standard treatment option for patients with advanced HR-positive, human epidermal growth factor receptor 2 (HER2)negative breast cancer [6]. Three selective CDK 4/6 inhibitors (palbociclib, ribociclib, abemaciclib) have significantly improved progression-free survival (PFS) and overall survival (OS) in patients with HRpositive advanced breast cancer and have been approved by the United States Food and Drug Administration (FDA) [7-10]. In the phase III PALOMA-2 trial, palbociclib plus letrozole showed significant longer PFS compared to endocrine alone in HR-positive, HER2-negative advanced breast cancer [10]. In another phase III PALOMA-3 trial, patients who received palbociclib plus fulvestrant had longer median PFS and OS compared to those who received placebo plus fulvestrant $[11,12]$.

Asian have younger age of breast cancer onset (45 to 50 years old) compared to Western population (55 to 60 years) $[13,14]$. In Korea, the median age at the time of breast cancer diagnosis is increasing, but 
still the highest incidence is seen at the age of 40-49 [15]. While palbociclib has been approved by the US FDA since 2015 , there are paucity of data on the real-world efficacy of palbociclib, especially in young Asian patients [16]. The purpose of this study is to evaluate the real-world efficacy and toxicity of palbociclib plus endocrine therapy in Korean patients with advanced breast cancer. This study included relatively younger patients compared to previous performed clinical trials.

\section{Method}

\section{Study design and population}

This study is a retrospective study performed in two tertiary referral hospitals in Korea [Seoul National University Hospital (SNUH, Seoul, Korea) and Seoul National University Bundang Hospital (SNUBH, Gyeonggi-do, Korea)]. Two hundred and sixteen breast cancer patients who received 1 st line palbociclib plus endocrine therapy between August 2016 and May 2019 were included. Patients were excluded if they received previous systemic chemotherapy for advanced/metastatic disease. Neoadjuvant and adjuvant chemotherapy, and adjuvant endocrine therapy were allowed. Patients with histologically confirmed HRpositive and HER2-negative breast cancer were included.

All patients received $125 \mathrm{mg}$ of palbociclib per day administered orally in 4-week cycles (3 weeks of treatment followed by 1 week off) combined with endocrine therapy. Dose reductions of palbociclib were allowed $(100 \mathrm{mg}$ or $75 \mathrm{mg}$ ) on the discretion of treating physician. Palbociclib was combined with one of three endocrine therapies (letrozole, exemestane, or fulvestrant). Letrozole was administered orally at a dose of $2.5 \mathrm{mg}$ per day, exemestane was administered orally at a dose of $25 \mathrm{mg}$ per day, and fulvestrant was administered by intramuscular injection at a dose of $500 \mathrm{mg}$ at day 1 and 15,29 , then once monthly thereafter.

Eligible patients were identified from the electronic database and medical charts were identified from the electronic database medical record system of SNUH and SNUBH. The study protocol was reviewed and approved by the institutional review board of SNUH [H-1904-025-1024] and SNUBH [B-2006/616 - 405]. This study was carried out in accordance with the recommendations of the Declaration of Helsinki for biomedical research involving human subjects.

\section{Analysis Of Tumor Subtype}

Immunohistochemical (IHC) staining of estrogen receptor (ER), progesterone receptor (PR), and HER-2 was performed with tumor specimen. HER2 fluorescence in situ hybridization was performed in patients with HER-2 IHC grade 2. Nuclear expression of tumor cells was interpreted as positive for ER and PR, while membrane staining of tumor cells was considered positive for HER2. ER and PR expression was categorized as positive when $\geq 1 \%$ of the tumor cells were stained [17]. HR was defined positive when either ER or PR was expressed. The intrinsic subtypes were defined by the 2013 St. Gallen Consensus Recommendations [18]. HR-positive, HER2-negative and Ki-67 $<14 \%$ were defined as luminal A. HR- 
positive, HER2-negative and Ki-67 $\geq 14 \%$ were considered luminal B [19]. Germline BRCA 1/2 mutation was tested based on the polymerase chain reaction and gene sequencing [20].

Primary endocrine resistance was defined as a relapse which occurred during the first 2 years of adjuvant endocrine therapy. Secondary or acquired endocrine resistance was defined when there was a relapse while on adjuvant endocrine therapy but after the first 2 years or within 12 months of completing adjuvant endocrine therapy [21].

\section{Statistical analysis}

The primary endpoint of this study was to investigate the real-world efficacy of palbociclib in terms of PFS. PFS was defined as the time from the start of palbociclib to radiologically or clinically disease progression, or death from any cause, whichever occurred first. Secondary outcomes were objective response rates (ORR), according to RECIST criteria version 1.1 and hematologic adverse events.

Categorical variables were compared using the chi-square test and continuous variables were compared using the independent-samples $T$ test. Missing data were not imputed. Kaplan-Meier method was used to obtain estimates of median PFS, and comparisons were made using the log-rank tests. Two-sided $p$ values of less than 0.05 were considered statistically significant. Univariate and multivariate proportional hazards regression models were used to identify independent risk factors for survival by means of the Cox proportional hazards regression models. Factors associated with prognosis were included in the multivariate analysis and forward stepwise methods were used to eliminate non-significant variables. Statistical analyses were performed using IBM SPSS statistics version 25 (SPSS, Chicago, IL, USA).

\section{Results}

\section{Patient characteristics}

A total of 216 breast cancer patients who were treated with palbociclib plus endocrine therapy were included. Two hundred and five (94.9\%) were treated palbociclib plus letrozole, 7 (3.2\%) received palbociclib plus exemestane, and 4 (1.9\%) received palbociclib plus fulvestrant. Baseline characteristics are summarized in Table 1. All patients were female with a median age of 56 (range: 29-89) years. Among 75 patients (34.7\%) who were premenopausal at the time of diagnosis of advanced breast cancer, 71 received bilateral oophorectomy prior to palbociclib treatment and 4 received gonadotropin-releasing hormone agonist ( $\mathrm{GnRHa}$ ) with palbociclib treatment. All patients had histologically confirmed breast cancer with HR-positive and HER2-negative. According to St. Gallen molecular subtype, $132(62.9 \%)$ had luminal A disease, and 78 (37.1\%) had luminal B disease. Seventy-three (33.8\%) patients were diagnosed with de novo stage IV disease and $143(66.2 \%)$ had relapsed disease. Among 143 relapsed patients, 131 (91.6\%) had received adjuvant endocrine therapy and 12 (8.4\%) did not receive adjuvant endocrine therapy. Based on adjuvant endocrine therapy, 58 (44.3\%) were endocrine sensitive, 21 (16.0\%) were 
primary resistance, and $50(38.2 \%)$ had secondary resistance. Thirty-three $(15.3 \%)$ patients were tested for germline BRCA1 and BRCA2 mutation status. Six (18.2\%) had germline BRCA1 or 2 mutation. 
Table 1

Baseline characteristics

\begin{tabular}{|c|c|c|c|c|c|c|c|}
\hline & \multicolumn{2}{|c|}{$\begin{array}{l}\text { Overall }(n= \\
216)\end{array}$} & \multicolumn{2}{|c|}{$\begin{array}{l}\text { Luminal A }(n=132 \text {, } \\
61.1 \%)\end{array}$} & \multicolumn{2}{|c|}{$\begin{array}{l}\text { Luminal B }(n=78 \text {, } \\
36.1 \%)\end{array}$} & \multirow[t]{2}{*}{$\begin{array}{l}P \\
\text { value }\end{array}$} \\
\hline & $\mathbf{N}$ & $\%$ & $\mathbf{N}$ & $\%$ & $\mathbf{N}$ & $\%$ & \\
\hline \multicolumn{8}{|l|}{ Age } \\
\hline$<50$ years & 73 & 33.8 & 49 & 37.1 & 23 & 29.5 & 0.26 \\
\hline$\geq 50$ years & 143 & 66.2 & 83 & 62.9 & 55 & 39.9 & \\
\hline \multicolumn{8}{|l|}{ Estrogen receptor } \\
\hline Positive & 216 & 100.0 & 132 & 100.0 & 57 & 74.0 & \\
\hline Negative & 0 & 0.0 & 0 & 0.0 & 20 & 26.0 & \\
\hline \multicolumn{8}{|c|}{ Progesteron receptor $(n=215)$} \\
\hline Positive & 171 & 79.5 & 109 & 82.6 & 57 & 74.0 & 0.14 \\
\hline Negative & 44 & 20.5 & 23 & 17.4 & 20 & 26.0 & \\
\hline \multicolumn{8}{|c|}{ Nucleic grade $(n=125)$} \\
\hline$H$ & 72 & 57.6 & 57 & 62.0 & 12 & 40.0 & 0.035 \\
\hline III & 53 & 42.4 & 35 & 38.0 & 18 & 60.0 & \\
\hline \multicolumn{8}{|c|}{ Histologic grade $(n=120)$} \\
\hline$H$ & 77 & 61.2 & 59 & 67.8 & 14 & 48.3 & 0.059 \\
\hline III & 43 & 35.8 & 28 & 32.2 & 15 & 51.7 & \\
\hline \multicolumn{8}{|l|}{ Disease presentation } \\
\hline De novo stage IV & 73 & 33.8 & 43 & 32.6 & 28 & 35.9 & 0.62 \\
\hline Relapsed disease & 143 & 66.2 & 89 & 67.4 & 50 & 64.0 & \\
\hline \multicolumn{8}{|c|}{ Adjuvant endocrine therapy $(n=143)$} \\
\hline No & 12 & 8.4 & 7 & 7.9 & 5 & 10.0 & 0.67 \\
\hline Yes & 131 & 91.6 & 82 & 92.1 & 45 & 90.0 & \\
\hline \multicolumn{8}{|c|}{ Endocrine resistance $(n=131)$} \\
\hline Endocrine sensitive & 58 & 44.3 & 37 & 41.6 & 17 & 35.4 & 0.53 \\
\hline
\end{tabular}

Abbreviations: N, number; OFS, ovarian function suppression; BSO, bilateral salphingo-oophorectomy; $\mathrm{GnRHa}$, Gonadotropin-releasing hormone agonist; 


\begin{tabular}{|c|c|c|c|c|c|c|c|}
\hline & \multicolumn{2}{|c|}{$\begin{array}{l}\text { Overall }(n= \\
216)\end{array}$} & \multicolumn{2}{|c|}{$\begin{array}{l}\text { Luminal A }(n=132 \text {, } \\
61.1 \%)\end{array}$} & \multicolumn{2}{|c|}{$\begin{array}{l}\text { Luminal B }(n=78 \text {, } \\
36.1 \%)\end{array}$} & \multirow[t]{2}{*}{$\begin{array}{l}P \\
\text { value }\end{array}$} \\
\hline & $\mathbf{N}$ & $\%$ & $\mathbf{N}$ & $\%$ & $\mathbf{N}$ & $\%$ & \\
\hline Primary resistance & 21 & 16.0 & 11 & 12.4 & 10 & 20.8 & \\
\hline Secondary resistance & 50 & 38.2 & 34 & 38.2 & 16 & 33.3 & \\
\hline \multicolumn{8}{|c|}{ Partner endocrine therapy } \\
\hline Letrozole & 205 & 94.9 & 124 & 93.9 & 76 & 97.4 & 0.50 \\
\hline Exemestane & 7 & 3.2 & 5 & 3.8 & 1 & 1.3 & \\
\hline Fulvestrnat & 4 & 1.9 & 3 & 2.3 & 1 & 1.3 & \\
\hline \multicolumn{8}{|l|}{ Menopausal status } \\
\hline $\begin{array}{l}\text { Natural } \\
\text { postmenopausal }\end{array}$ & 141 & 65.3 & 84 & 63.6 & 52 & 66.7 & 0.90 \\
\hline OFS using BSO & 71 & 32.9 & 46 & 34.8 & 25 & 32.1 & \\
\hline OFS using $\mathrm{GnRHa}$ & 4 & 1.9 & 2 & 1.5 & 1 & 1.3 & \\
\hline \multicolumn{8}{|c|}{ Germline BRCA $1 / 2$ mutation } \\
\hline Positive & 6 & 2.8 & 3 & 2.3 & 3 & 3.8 & 0.34 \\
\hline Negative & 27 & 12.5 & 19 & 14.4 & 8 & 10.3 & \\
\hline Test was not done & 183 & 84.7 & 110 & 83.3 & 67 & 85.9 & \\
\hline \multicolumn{8}{|l|}{ Metastatic site } \\
\hline Bone & 132 & 61.1 & 76 & 57.6 & 52 & 66.7 & 0.19 \\
\hline Liver & 32 & 14.8 & 19 & 14.4 & 13 & 16.7 & 0.66 \\
\hline Lung & 85 & 39.4 & 55 & 41.7 & 27 & 34.6 & 0.31 \\
\hline Brain & 5 & 2.3 & 3 & 2.3 & 2 & 2.6 & 0.89 \\
\hline
\end{tabular}

\section{Real-world Efficacy Of Palbociclib Plus Endocrine Therapy}

After a median follow-up duration of 16.0 months, 75 progression events and 11 deaths have occurred. The median PFS of palbociclib plus endocrine therapy was 33.0 months (95\% confidence interval [Cl] 24.7 to 41.3 ). Among 123 (56.9\%) patients who had measurable disease, there was no complete 
response, 73 (59.3\%) had partial response, 31 (25.2\%) had stable disease, and 19 (15.4\%) had progressive disease. The ORR was $59.3 \%$ (Table 2).

Table 2

Objective response rate

\begin{tabular}{|lll|}
\hline & Response rate, \% & P-value \\
\hline Objective response rate $(\mathrm{n}=123)$ & 59.3 & \\
\hline Intrinsic subtype & & 0.19 \\
\hline Luminal A & 62.0 & \\
\hline Luminal B & 58.3 & \\
\hline Age & & \\
\hline$<50$ years & 72.7 & \\
\hline$\geq 50 y e a r s$ & 67.1 & 0.23 \\
\hline Menopausal state & & \\
\hline Natural postmenopausal & 54.5 & 0.19 \\
\hline OFS using BSO or GnRH agonist & 71.4 & \\
\hline Germline BRCA 1/2 mutation & & \\
\hline Positive & 0.0 & \\
\hline Negative & 60.0 & \\
\hline Abbreviations: N, number; & & \\
\hline
\end{tabular}

We next evaluated whether baseline characteristics may affect palbociclib efficacy. Patient age or menopausal status did not affect treatment response. There was no difference in PFS (not reached $v s$. 33.0 months, $p=0.56)$ and ORR $(72.7 \%$ vs. $67.1 \%, p=0.11)$ (Table 2$)$ according patients age. Similarly, menopausal status did not affect PFS (33.0 months vs. not reached, $p=0.82)$ or ORR $(54.5 \% v s .71 .4 \%, p$ $=0.23$ ) (Table 2). However, molecular tumor subtype was associated with palbociclib response. Luminal A patients had longer PFS compared to luminal B patients (Not reached $v s .33 .0$ months, $p=0.019$ ) (Fig. 1). In addition, there was a tendency of higher ORR in luminal A patients compared to luminal $B$ patients $(62.0 \%$ vs. $58.3 \%, p=0.19)$ (Table 2$)$. Among 33 patients who had germline $B R C A 1 / 2$ mutation test, $6(18.2 \%)$ had germline $B R C A 1 / 2$ mutation. Patients with germline $B R C A 1 / 2$ mutation had shorter PFS compared to germline $B R C A$ wild type patients (9.0 months $v s$. not reached, $p=0.031)$ and patients who were not tested for germline BRCA status (9.0 months $v s$. 33.0 months, $p=0.001$ ) (Fig. 2). PFS was similar between germline BRCA wild type patients and those who did not have germline BRCA test (Not reached vs. 33.0 months, $p=0.54$ ) (Fig. 2). 
To adjust for baseline characteristics, we performed multivariable analysis with a Cox proportional hazard model. Due to limited number of patients who had germline BRCA status and low incidence of germline $B R C A 1 / 2$ mutation, patients who did not test germline $B R C A$ status was combined with germline $B R C A$ wild type in the multivariate analysis. Multivariate analysis revealed nuclear grade, intrinsic subtype, and germline BRCA $1 / 2$ mutation as an independent prognostic factor for PFS (Table 3). Luminal B subtype (adjusted hazard ratio 1.90, 95\% Cl 1.04-3.47, $p=0.038$ ) and germline $B R C A 1 / 2$ mutation (adjusted hazard ratio $5.57,95 \% \mathrm{Cl} 1.91-16.24, p=0.002$ ) were independent poor prognostic factors for PFS.

Table 3

Multivariate analysis of progression free survival

\begin{tabular}{|c|c|c|}
\hline & Adjusted HR (95\% Cl) & $P$-value \\
\hline Nucleic grade & $2.09(1.16,3.77)$ & 0.014 \\
\hline \multicolumn{3}{|l|}{ Intrinsic subtype } \\
\hline Luminal A & 1 & 0.038 \\
\hline Luminal B & $1.90(1.04,3.47)$ & \\
\hline \multicolumn{3}{|c|}{ Germline BRCA $1 / 2$ mutation } \\
\hline Negative or not tested & 1 & 0.002 \\
\hline Positive & $5.57(1.91,16.24)$ & \\
\hline
\end{tabular}

The mean time to objective response was 3.8 months. There was no significant difference in mean time to objective response between luminal $\mathrm{A}$ and luminal B (3.9 vs. 3.6 months, $p=0.32)$. The mean duration of objective response was 15.1 months. Tumor subtype did not impact mean duration of response (16.5 months in luminal A vs. 13.1 months in luminal $B, p=0.37$ ).

\section{Adverse Events}

The most common hematologic adverse events were neutropenia. Among 216 patients, 210 (97.2\%) reported adverse events with any grade of neutropenia, and $182(86.7 \%)$ reported events of grade 3 or higher grade neutropenia. And 7 patients (3.2\%) reported events of neutropenic fever. Other hematologic adverse events included anemia and thrombocytopenia. Sixty (34.5\%) showed grade 3 or 4 anemia and 7 $(8.8 \%)$ showed grade 3 or 4 thrombocytopenia. There was no significant difference in neutropenia according to age at $50(86.3 v s .83 .2 \%, p=0.47)$.

\section{Discussion}


In recent years, CDK 4/6 inhibitors have changed the management of advanced HR-positive, HER2negative breast cancer. There are ethnic differences in the prevalence of breast cancer that Asian countries have relatively younger age of onset compared to Western. There are yet paucity of data on the real-world efficacy of CDK 4/6 inhibitor in young Asian population. This study was conducted to investigate the real-world efficacy of palbociclib plus endocrine therapy in Asian women. We revealed that the efficacy of palbociclib plus endocrine therapy in young Asian women is comparable to that of previous conducted phase III clinical trials.

The phase III PALOMA-2 trial evaluated the efficacy of 1 st line palbociclib plus letrozole in postmenopausal women with HR-positive, HER2-negative advanced breast cancer [10]. Palbociclibletrozole group had higher ORR (55.3 vs. 44.4\%, $p=0.03)$ and improved PFS (27.6 vs. 14.5 months, $p<$ $0.0001)$ compared to placebo-letrozole group $[10,22]$. In PALOMA-2 trial, PFS in patients over 65 was similar to that of patients under 65 (hazard ratio for disease progression or death, 0.57) [10]. In the phase III PALOMA-3 study, women with HR-positive, HER2-negative advanced breast cancer who had progressed on previous endocrine therapy were enrolled [11]. Patients were randomly assigned to palbociclib plus fulvestrant group or placebo plus fulvestrant group. Patients who received palbociclib plus fulvestrant had improved ORR (10.4 vs. 6.3\%, $p=0.16)$, PFS (9.2 vs. 3.8 months, $p<0.001)$, and OS (34.9 vs. 28.0 months, $p=0.09$ ) compared to those who receive placebo plus fulvestrant. Twenty percentage of patients enrolled in the PALOMA-3 trial was pre-/peri-menopausal and menopausal status did not affect PFS or OS $[11,12]$.

In this study, we have evaluated the real-world efficacy of 1 st line palbociclib plus endocrine therapy in advanced or metastatic breast cancer patients. Palbociclib plus endocrine therapy showed a median PFS of 33.0 months ( $95 \% \mathrm{Cl} 24.7$ to 41.3 ) with an ORR of $59.3 \%$, which is similar to that of PALOMA-2 trial. All patients included in the present study were Asian who has younger age of breast cancer onset. The median age of our study was 53 years old which is younger than that of PALOMA-2 trial (62 years old). In line with previous conducted trials, patient age or menopausal status did not affect PFS or ORR of palbociclib plus endocrine therapy. However, St. Gallen intrinsic subtype was a significant prognostic factor. Multivariate analysis revealed Luminal $B$ as an independent negative prognostic factor for PFS (adjusted hazard ratio 1.90, $p=0.038$ ). It is known that while luminal A tumors have indolent feature and are sensitive to antiestrogen, luminal $B$ tumors often show rapid progression and resistance to endocrine therapy [23]. Aggressive nature and endocrine resistant feature may have resulted in the poor PFS and ORR of luminal B tumors. Recent meta-analysis data show that CDK 4/6 inhibitor plus endocrine therapy is non-inferior to conventional chemotherapy in terms of PFS and time to progression [24]. In addition, CDK 4/6 inhibitor plus endocrine therapy showed similar ORR compared to conventional chemotherapy. As CDK 4/6 inhibitor plus endocrine therapy have superior toxicity profiles and drug administration route compared to conventional chemotherapy, it is the preferred treatment option in HR-positive, HER2negative breast cancer patients. However, in the present study, luminal B patients had shorter PFS and tendency of low ORR compared to luminal A patients. As such, CDK 4/6 inhibitor plus endocrine therapy should be used in caution in Luminal B patients with visceral disease. 
Another important result of our study is that we showed potential negative prognostic role of germline $B R C A 1 / 2$ mutation. Patients with germline BRCA 1/2 mutation had a worse PFS compared to patients with germline $B R C A$ wild type and those who did not test germline $B R C A$ status. Multivariate analysis revealed germline $B R C A 1 / 2$ mutation as an independent negative prognostic factor for PFS compared to patients with germline $B R C A$ wild type or those who did not have germline $B R C A$ test (adjusted hazard ratio $5.57,95 \% \mathrm{Cl} 1.91-16.24, p=0.002$ ). Although there are limited number of patients with germline $B R C A 1 / 2$ mutation, this is in line with previous studies showing poor outcome of $B R C A 1 / 2$ mutation patients treated with endocrine therapy plus CDK 4/6 inhibitors [25]. We believe future study comparing frontline poly adenosine diphosphate ribose polymerase inhibitor with CDK 4/6 inhibitors in HR-positive breast cancer with germline $B R C A 1 / 2$ mutation will provide valuable information.

There are some limitations in the present study. The tumor specimens we used to identify tumor subtype was acquired in various time point. It would be ideal to obtain tissue prior to CDK 4/ 6 inhibitor treatment, but unfortunately, most samples were acquired at initial breast cancer diagnosis. Another limitation of the present study is that we did not have enough data on germline BRCA 1/2 mutation status or other genetic characteristics to draw answer on the prognostic role of molecular findings. However, the strength of our study is that our study was relatively homogeneous in terms of ethnic and that all patients received topof-the-line medical care in a high-volume tertiary referral hospital.

\section{Conclusions}

In conclusion, the efficacy and toxicity of palbociclib in the real-world was comparable to those of previous conducted phase III clinical trials. In addition, palbociclib with endocrine therapy was an effective treatment option for young Asian breast cancer patients. However, as the ORR and PFS is inferior in luminal $B$ subtype, $C D K 4 / 6$ inhibitor plus endocrine therapy should be used in caution in luminal $B$ patients with visceral disease.

\section{List Of Abbreviations}

CDK

Cyclin dependent kinase

Cl

Confidence interval

ER

Estrogen receptor

FDA

Food and Drug Administration

\section{GnRHa}

Gonadotropin-releasing hormone agonist

HER2

Human epidermal growth factor receptor 2 
HR

Hormone receptor

IHC

Immunohistochemical

ORR

Overall response rate

os

Overall survival

PFS

Progression-free survival

PR

Progesterone receptor

SNUH

Seoul National University Hospital

SNUBH

Seoul National University Bundang Hospital

\section{Declarations}

\section{Ethics approval and consent to participate}

The study protocol was reviewed and approved by the institutional review board of SNUH [H-1904-0251024] and SNUBH [B-2006/616-405].

\section{Consent for publication}

All authors gave consent for the publication of the manuscript in Breast Cancer Research.

\section{Availability of supporting data}

The datasets used and/or analysed during the current study are available from the corresponding author on reasonable request

\section{Completing interests}

The authors declare that they have no competing interests.

\section{Funding}

This work was supported by the National Research Foundation of Korea(NRF) grant funded by the Korea government(MSIT). (No. NRF2020R1A2C301088311)

\section{Acknowledgements}


This work was supported by the National Research Foundation of Korea(NRF) grant funded by the Korea government(MSIT). (No. NRF2020R1A2C301088311)

Author information

Song Yi Park and Koung Jin Suh are co-first authors

\section{Affiliations}

Department of Internal Medicine, Seoul National University Hospital, 101 Daehang-Ro, Jongno-Gu, Seoul, 03080, South Korea

Song Yi Park, Dae-Won Lee, Miso Kim, Kyung-Hun Lee, Tae-Yong Kim \& Seock-Ah Im

Department of Internal Medicine, Seoul National University Bundang Hospital, 82, Gumi-ro 173 Beon-gil, Bundang-gu, Seongnam-si, Gyeonggi-do 13620, South Korea

Koung Jin Suh, Se Hyun Kim \& Jee Hyun Kim

Department of Translational Medicine, Seoul National University, 103 Daehang-Ro, Jongno-Gu, Seoul, 03080, South Korea

Koung Jin Suh, Dae-Won Lee, Se Hyun Kim, Jee Hyun Kim \& Seock-Ah Im

Department of Pathology, Seoul National University Hospital, 101 Daehang-Ro, Jongno-Gu, Seoul, 03080, South Korea

Han Suk Ryu \& In Ae Park

Cancer Research Institute, Seoul National University, 101 Daehang-Ro, Jongno-Gu, Seoul, 03080, South Korea

Kyung-Hun Lee, Tae-Yong Kim \& Seock-Ah Im

\section{Contributions}

DWL and SAI contributed to the study concept and design. SYP and KJS contributed to the acquisition of the data. All authors contributed to the analysis and interpretation of data, and drafting/revision of the article. All authors read and approved the final manuscript.

Corresponding author

Co-correspondence to Dae-Won Lee and Seock-Ah Im

\section{References}


1. Bray F, Ferlay J, Soerjomataram I, Siegel RL, Torre LA, Jemal A. Global cancer statistics 2018 : GLOBOCAN estimates of incidence and mortality worldwide for 36 cancers in 185 countries. Cancer J Clin. 2018;68(6):394-424.

2. Jung K-W, Won Y-J, Kong H-J, Lee ES. Prediction of Cancer Incidence and Mortality in Korea, 2019. Cancer Res Treat. 2019;51(2):431-7.

3. Althuis MD, Dozier JM, Anderson WF, Devesa SS, Brinton LA. Global trends in breast cancer incidence and mortality 1973-1997. Int J Epidemiol. 2005;34(2):405-12.

4. Jung KW, Won YJ, Kong HJ, Lee ES. Cancer Statistics in Korea: Incidence, Mortality, Survival, and Prevalence in 2015. Cancer Res Treat. 2018;50(2):303-16.

5. Setiawan VW, Monroe KR, Wilkens LR, Kolonel LN, Pike MC, Henderson BE. Breast cancer risk factors defined by estrogen and progesterone receptor status: the multiethnic cohort study. Am J Epidemiol. 2009;169(10):1251-9.

6. Finn RS, Aleshin A, Slamon DJ. Targeting the cyclin-dependent kinases (CDK) 4/6 in estrogen receptor-positive breast cancers. Breast cancer research: BCR. 2016;18(1):17.

7. Hamilton E, Infante JR. Targeting CDK4/6 in patients with cancer. Cancer treatment reviews. 2016;45:129-38.

8. Im S-A, Lu Y-S, Bardia A, Harbeck N, Colleoni M, Franke F, Chow L, Sohn J, Lee K-S, Campos-Gomez S, et al. Overall Survival with Ribociclib plus Endocrine Therapy in Breast Cancer. N Engl J Med. 2019;381(4):307-16.

9. Goetz MP, Toi M, Campone M, Sohn J, Paluch-Shimon S, Huober J, Park IH, Trédan O, Chen SC, Manso L, et al. MONARCH 3: Abemaciclib As Initial Therapy for Advanced Breast Cancer. Journal of clinical oncology: official journal of the American Society of Clinical Oncology. 2017;35(32):363846.

10. Finn RS, Martin M, Rugo HS, Jones S, Im S-A, Gelmon K, Harbeck N, Lipatov ON, Walshe JM, Moulder S, et al. Palbociclib and Letrozole in Advanced Breast Cancer. N Engl J Med. 2016;375(20):1925-36.

11. Turner NC, Ro J, André F, Loi S, Verma S, Iwata H, Harbeck N, Loibl S, Huang Bartlett C, Zhang K, et al. Palbociclib in Hormone-Receptor-Positive Advanced Breast Cancer. N Engl J Med. 2015;373(3):20919.

12. Turner NC, Slamon DJ, Ro J, Bondarenko I, Im S-A, Masuda N, Colleoni M, DeMichele A, Loi S, Verma $\mathrm{S}$, et al. Overall Survival with Palbociclib and Fulvestrant in Advanced Breast Cancer. N Engl J Med. 2018;379(20):1926-36.

13. Saika K, Sobue T. Epidemiology of breast cancer in Japan and the US. Japan Medical Association Journal. 2009;52:39-44.

14. Youlden DR, Cramb SM, Yip CH, Baade PD. Incidence and mortality of female breast cancer in the Asia-Pacific region. Cancer Biol Med. 2014;11(2):101-15.

15. Kang SY, Kim YS, Kim Z, Kim HY, Lee SK, Jung KW, Youn HJ. Basic Findings Regarding Breast Cancer in Korea in 2015: Data from a Breast Cancer Registry. Journal of breast cancer. 2018;21(1):1-10. 
16. https://www.fda.gov/drugs/resources-information-approved-drugs/palbociclib-ibrance.

17. Hammond ME, Hayes DF, Dowsett M, Allred DC, Hagerty KL, Badve S, Fitzgibbons PL, Francis G, Goldstein NS, Hayes M, et al. American Society of Clinical Oncology/College Of American Pathologists guideline recommendations for immunohistochemical testing of estrogen and progesterone receptors in breast cancer. Journal of clinical oncology: official journal of the American Society of Clinical Oncology. 2010;28(16):2784-95.

18. Prat A, Pineda E, Adamo B, Galván P, Fernández A, Gaba L, Díez M, Viladot M, Arance A, Muñoz M. Clinical implications of the intrinsic molecular subtypes of breast cancer. Breast. 2015;24(Suppl 2):26-35.

19. Goldhirsch A, Winer EP, Coates AS, Gelber RD, Piccart-Gebhart M, Thürlimann B, Senn HJ. Personalizing the treatment of women with early breast cancer: highlights of the St Gallen International Expert Consensus on the Primary Therapy of Early Breast Cancer 2013. Annals of oncology: official journal of the European Society for Medical Oncology. 2013;24(9):2206-23.

20. Richards S, Aziz N, Bale S, Bick D, Das S, Gastier-Foster J, Grody WW, Hegde M, Lyon E, Spector E, et al. Standards and guidelines for the interpretation of sequence variants: a joint consensus recommendation of the American College of Medical Genetics and Genomics and the Association for Molecular Pathology. Genetics in medicine: official journal of the American College of Medical Genetics. 2015;17(5):405-24.

21. Jung K-W, Won Y-J, Kong H-J, Lee ES. Cancer Statistics in Korea: Incidence, Mortality, Survival, and Prevalence in 2015. Cancer Res Treat. 2018;50(2):303-16.

22. Rugo HS, Finn RS, Diéras V, Ettl J, Lipatov O, Joy AA, Harbeck N, Castrellon A, lyer S, Lu DR, et al: Palbociclib plus letrozole as first-line therapy in estrogen receptor-positive/human epidermal growth factor receptor 2-negative advanced breast cancer with extended follow-up. Breast cancer research and treatment 2019, 174(3):719-729.

23. Sotiriou C, Pusztai L. Gene-Expression Signatures in Breast Cancer. N Engl J Med. 2009;360(8):790800.

24. Schettini F, Giuliano M, Rognoni C, De Placido S, Arpino G, Milani M, Giordano A, Cristofanilli M, Jerusalem G, Bachelot T, et al: Abstract P1-16-01: Efficacy of endocrine- versus chemotherapy-based treatments in hormone receptor-positive (HR + ve), HER2-negative (HER2-ve) postmenopausal metastatic breast cancer (mBC): A network meta-analysis (NMA). Cancer research 2019, 79(4 Supplement):P1-16-01-P11-16-01.

25. Frenel JS, Dalenc F, Pistilli B, de La Motte Rouge T, Levy C, Mouret-Reynier MA, Hardy-Bessard AC, Bonichon-Lamichhane N, Greilsamer C, Delecroix V, et al. ESR1 mutations and outcomes in BRCA1/2 or PALB2 germline mutation carriers receiving first line aromatase inhibitor + palbociclib $(A I+P)$ for metastatic breast cancer (MBC) in the PADA-1 trial. Ann Oncol. 2020;304P:31:S364.

\section{Figures}




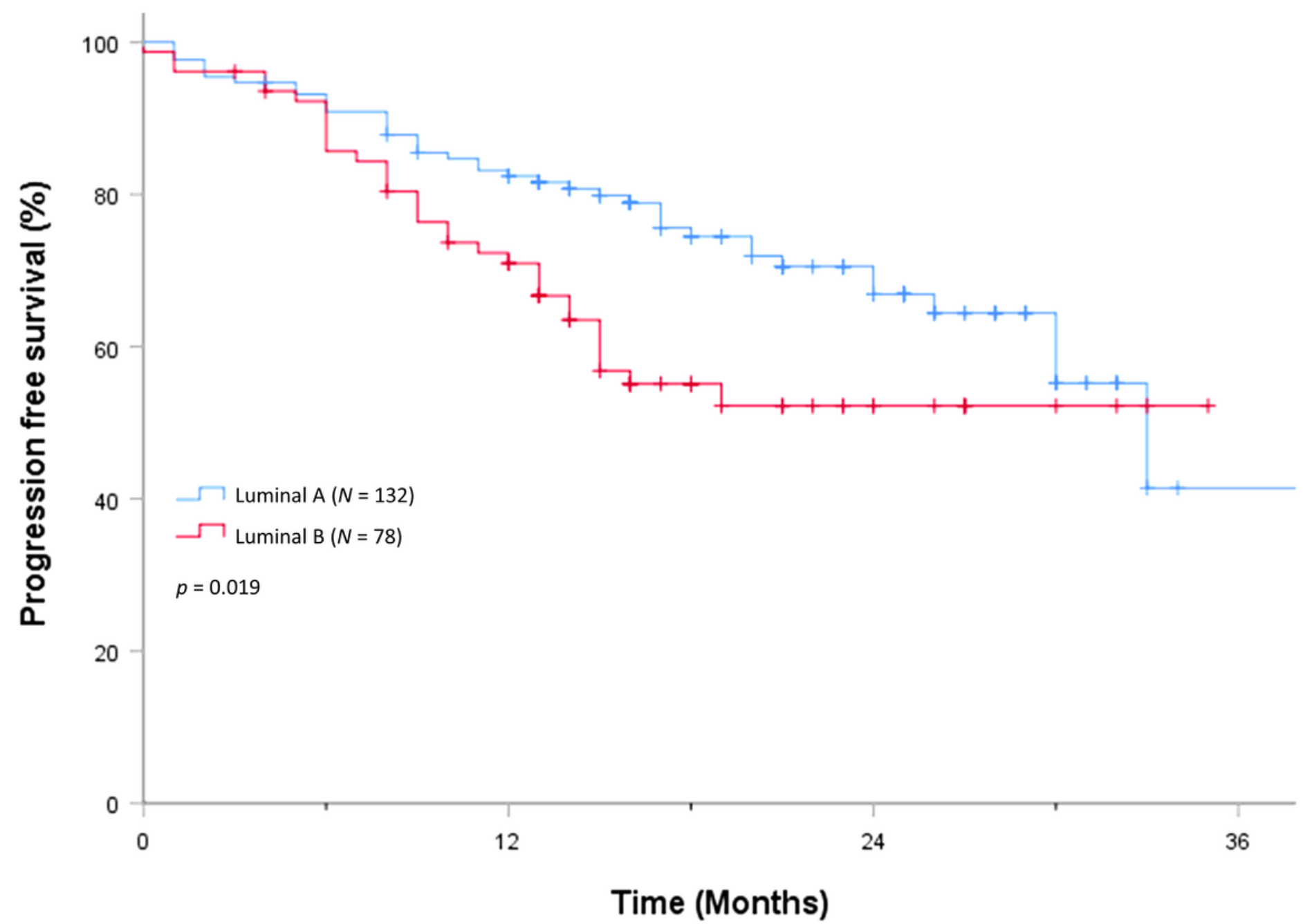

Figure 1

Progression free survival according to St.Gallen molecular subtype Abbreviations: PFS, progression free survival. 


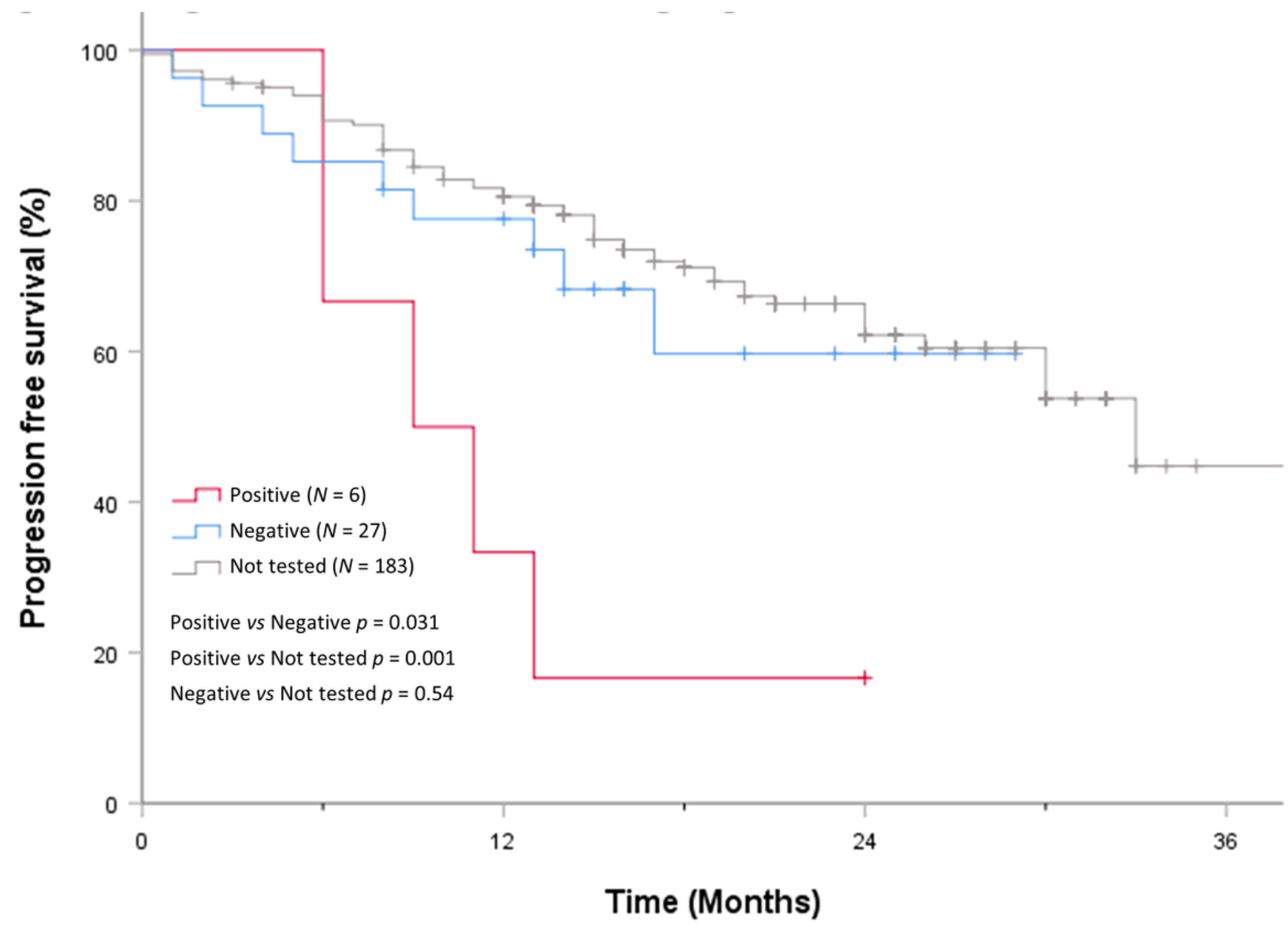

Figure 2

Progression free survival according to germline BRCA 1/2 mutation status Abbreviations: PFS, progression free survival; 